\title{
GEOGRAPHICAL ROUTING PROTOCOLS FOR MOBILE AD HOC NETWORKS- A SURVEY ON THEIR PERFORMANCE ANALYSIS
}

\author{
Suma $\mathbf{R}^{1}$, B G Premasudha ${ }^{2}$ \\ ${ }^{1}$ Associate Professor, Department of MCA, Sri Siddhartha Institute of Technology, Karnataka, India, \\ sumaraviram@gmail.com \\ ${ }^{2}$ Professor, Department of MCA, Siddaganga Institute of Technology, Karnataka, India \\ bgpremasudha@gmail.com
}

\begin{abstract}
Mobile Ad Hoc network is an autonomous system of mobile nodes connected by wireless links forming a temporary network without the aid of any centralized administration or infrastructure. Each node operates not only as an end system but also as a router to forward packets. The nodes are free to move themselves into a network. These networks have no fixed topology due to the high degree of node mobility. To accommodate the changing topology, special routing protocols are needed. The goal of the routing protocol is to have an efficient route establishment between a pair of nodes, so that messages can be delivered in a timely manner. Mobile Ad Hoc routing protocols are divided into Flat routing, Hierarchical routing, Geographical routing, Power aware routing and Multicast routing. It is difficult to determine which protocols may perform well under a number of different network scenarios. This paper provides an overview of geographical routing protocols proposed in the literature and performance comparison of geographical routing protocols.
\end{abstract}

Index Terms: Mobile Ad Hoc Network, Geographical Routing protocols.

\section{INTRODUCTION}

Mobile Ad Hoc network is self-organizing and dynamic $[1,2,3]$. Networks are formed on-the-fly, devices can leave and join the network during its lifetime. Devices in Mobile Ad Hoc network should be able to detect the presence of the other devices and perform the necessary set-up to facilitate communications and the sharing of data and services. An ad Hoc network consists of a set of mobile nodes that are connected by wireless links [1]. The network topology in such a network may keep changing randomly. Routing protocols that find a path to be followed by data packets from a source node to a destination node used in traditional wired networks cannot be directly applied in Mobile ad hoc wireless networks due to their highly dynamic topology, absence of established infrastructure for centralized administration, bandwidth constrained wireless links, and resource constrained nodes. A variety of routing protocols for wireless networks have been proposed. Routing in Mobile Ad Hoc network can be classified according to network structure as Flat routing, Hierarchical routing, Geographical routing, Power aware routing and Multicast routing.

This paper address the different geographical location based routing protocols in mobile ad hoc networks with their characteristics and complexity analysis. Since nodes in mobile ad hoc network can move randomly, the topology may change arbitrarily and frequently at unpredictable times. Transmission and reception parameters may also impact the topology. So it is very difficult to find and maintain an optimal route. The routing algorithm must react quickly to topological changes.

In this paper, Section 2 briefly describes the routing in mobile ad hoc networks. Section 3 explains the geographical location based routing protocols such as DREAM, LAR, GLS (Grid), GPSAL and ZHLS. Section 4 presents the basic characteristics and complexity comparison of geographical location based routing protocols. Section 5 focuses on summary of results and general factors of geographical location based routing protocols and Section 6 contains the conclusion.

\section{ROUTING IN MOBILE AD HOC NETWROKS}

Mobile Ad Hoc network with the collection of wireless mobile nodes form a temporary network without the aid of any stand-alone infrastructure or centralized administration [3]. Mobile Ad Hoc networks are self-configuring multi hop wireless networks with mobility of participating nodes. The structure of the network changes dynamically due to the mobility of the nodes [5]. Nodes in these networks utilize the same random access wireless channel and cooperative multi hop forwarding. The nodes in the network not only act as hosts but also as routers that route data to/from other nodes in the network [6]. 
There is no infrastructure support in this network; routing procedure is always needed to find a path to forward the packets appropriately between the source and the destination. Hence each node is able to forward data to other nodes. Additional problems are created along with the problems of dynamic topology due to the unpredictable connectivity changes $[7,8]$.

There are several well-known protocols $[9,10]$ in the literature that have been specifically developed to cope with the limitations imposed by mobile ad hoc networking environments. The following sections briefly present the different geographical location based routing protocols in Mobile Ad Hoc networks.

\section{GEOGRAPHICAL ROUTING}

Routing protocols in Mobile Ad Hoc networks can be classified in many ways [11]. Most of them are classified depending on routing strategy and network structure $[18,5]$. According to the routing strategy, the routing protocols can be categorized as table-driven and source initiated, while depending on the network structure these are classified as flat routing, hierarchical routing, geographical location based routing, power aware routing and multicast routing[12] shown in below Fig -1.

The goal of Geographical location-based routing protocol is to reach a specific host (as specified by an address or other identifier). However, as geographic routing is based on the coordinates, not the identifier, one can't directly reach the intended target without knowing that intended target's location. Thus, geographic routing must be augmented with a service that can translate identifiers into locations. The GPS system provides a scalable and elegant solution to this problem. Geographical location-based protocols make it possible to have larger networks without scalability problems.

Geographical location-based routing algorithms use position information for making packet forwarding decisions. They do not need to exchange and maintain routing information and work nearly stateless. This makes geographic routing attractive for wireless ad hoc and sensor networks. Most geographic routing algorithms use a greedy strategy that tries to approach the destination in each step, e.g. by selecting the neighbor closest to the destination as a next hop.

Geographic routing is a technique to deliver a message to a node in a network over multiple hops by means of position information. Routing decisions are not based on network addresses and routing tables; instead, messages are routed towards a destination location. With knowledge of the neighbors' location, each node can select the next hop neighbor that is closer to the destination, and thus advance towards the destination in each step. The fact that neither routing tables nor route discovery activities are necessary makes geographic routing attractive for dynamic networks such as wireless ad hoc and sensor networks. In such networks, acquiring and maintaining routing information is costly as it involves additional message transmissions that require energy and bandwidth and frequent updates in mobile and dynamic scenarios. In contrast, there are geographic routing algorithms that work nearly stateless and can provide high message delivery rates under mobility.

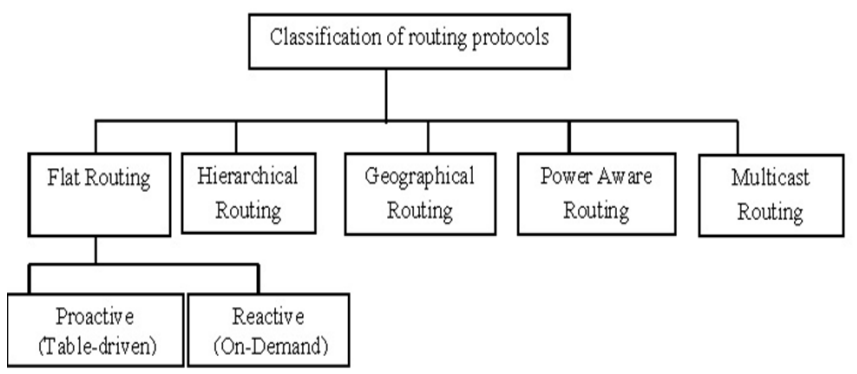

Fig-1: Classification of Routing Protocols in MANETS

\subsection{Geographic Routing Protocols}

The Geographical routing protocols imply that the hosts participating in the routing process should be aware of their geographic positions. An advantage of geographic routing protocols is that they prevent network-wide searches for destinations. Control and data packets can be sent in the general direction of the destination if the recent geographical coordinates are known. This reduces control overhead in the network. A disadvantage is that all nodes must have access to their geographical coordinates all the time to make the geographical routing protocols useful. The routing update must be done faster than the network mobility rate to make the location-based routing effective. This is because the nodes locations may change quickly in a mobile ad hoc network.

There are two approaches to geographic mobile ad hoc networks 1. Actual geographic coordinates (as obtained through GPS-the Global Positioning System). 2. Reference points in some fixed coordinate system.

\subsubsection{DREAM (Distance Routing Effect Algorithm for Mobility)}

DREAM [13] is a proactive, multi-path, location-aware routing protocol. DREAM makes use of the so-called distance effect to regulate the frequency of topological updates. According to the distance effect, the greater the distance between two nodes, the lower is their relative mobility. DREAM also makes use of the mobility rate of the nodes to regulate the frequency of location updates: the faster a node moves, the higher is the frequency of location updates from that node. A node records the locations of all its peer nodes in a location table. Using this location information, a node forwards the data packet to a set of neighbors that lie in the direction to the destination. If no such neighbors could be selected, the data packet is dropped. The destination responds with an acknowledgment (ACK) when it receives the data packet forwarded by a designated set of nodes. The ACK is forwarded to the source node in a fashion similar to that of the data packet. If the source node 
fails to receive an ACK through a designated set of nodes, it floods the data packet. Once at least one path between the source and destination are learnt, the source could start sending data packets using the learned paths, preferably the shortest hop path. The routing metric in DREAM has been referred to as shortest hop path in [18]. Hence, DREAM belongs to the class of protocols based on minimum-weight path based routing.

\subsubsection{LAR (Location-Aided Routing)}

Like DREAM, Location-Aided Routing (LAR) [14] is an example of restricted directional flooding routing protocols; however, partial flooding is used in LAR for path discovery purpose. Hence, LAR proposes the use of position information to enhance the route discovery phase of reactive Ad-Hoc routing approaches. The expected zone is fixed from the source based on the available position information. A request zone is defined as the set of nodes that should forward the route discovery packet. The request zone typically includes the expected zone. Two request zone schemes have been proposed. In Scheme 1 of LAR, a rectangular geographic region will be selected where nodes will forward the route discovery packet only if they are within that specific region. In Scheme 2 of LAR, the source or an intermediate node will forward the message to all nodes that are closer to the destination than itself. Thus, the node that receives the route request message will check if it is closer to the destination than the previous hop if so it will retransmit the route request message; otherwise, it will drop the message. In order to find the shortest path in the network level, instead of selecting a single node as the next hop, several nodes will be selected for managing the route request message and each of them will put its IP address in the header of the request packet. Therefore, the route through which the route request message is passed will be saved in the header of the message; message size will grow as it goes far from the source and the routing overhead will be increased.

\subsubsection{GLS(Grid)}

Grid [15] is a hierarchical location-aware routing protocol. The entire geographical area of the MANET is divided into logical grids each of size $\mathrm{d} * \mathrm{~d}$. Grids are identified using the conventional $(\mathrm{x}, \mathrm{y})$ co-ordinate system, while hosts have their own unique ids. Routing information is maintained in a grid-to-grid basis rather than the usual host-to-host manner. Each grid has a gateway node that (i) forwards route discovery requests to neighboring grids (ii) propagates data packets to neighboring grids and (iii) maintains routes passing through the grids. Non-gateway nodes in a grid do not forward packets. Nodes near the centre of the grid are preferred to be the gateway of the grid. Such a gatewayelection rule increases the probability of connectivity between grids. Route discovery procedure is similar to that employed in Ad Hoc On-Demand Distance Vector routing protocol AODV, the exception being the gateway nodes forward the route discovery RREQ packets and routes are maintained on a grid-to-grid basis. When a gateway node moves into a neighboring grid, the route could be still maintained by electing a new gateway node locally within the grid. The route discovery overhead is reduced drastically and routes generally fail, only when the source or destination moves out to a grid that is not the neighboring grid in the existing route. Hence, GRID could be grouped under the category of stability-based routing protocols. The grid size represents the trade-off between grid connectivity, route optimality and stability.

\subsubsection{GPSAL (GPS/Ant-Like Routing)}

In GPSAL (GPS/Ant-Like Routing Algorithm)[16] an ant agent has the responsibility of collecting and disseminating the information about the nodes' position. The software agents modeled on ants may follow different paths. In fact, the more different paths they follow the more nodes' positions are disseminated. These software agents are implemented as a packet transmitted from node to node until the destination node is reached and a response is sent back to the mobile unit that has created it. The GPSAL algorithm does not use flooding even though it provides a smaller overhead at higher speed.

\subsubsection{ZHLS (Zone Hybrid Link State)}

The network is divided into zones. Each node is assumed to know its location and hence be able to map a given location to its corresponding zone id. Two zones are assumed to be connected if at least one node in one zone is connected to a node in the other zone. Routing within and in between zones is based on shortest path routing. Hence, ZHLS [17] belongs to the category of routing protocols based on minimumweight path based routing.

\section{COMPARISION OF GEOGRAPHICAL LOCATION BASED ROUTING PROTOCOLS}

This section briefs all geographical location based routing protocols and lists the comparisons of basic characteristics and complexity of all of them. Below Table 4.1 provide the basic characteristics of geographical location based routing protocols. From this table we can understand the basic characteristics features of all the five geographical location based routing protocols. 
Table 4.1: Comparison of Basic characteristics of Geographical location based routing protocols.

\begin{tabular}{|l|l|l|l|l|l|}
\hline Protocol & $\begin{array}{l}\text { Routing } \\
\text { Structure }\end{array}$ & $\begin{array}{l}\text { Number of } \\
\text { routing tables }\end{array}$ & Frequency of updates & $\begin{array}{l}\text { Hello } \\
\text { Message }\end{array}$ & Characteristic feature \\
\hline DREAM & Flat & One & Mobility based & No & Controlled rate of updates by mobility and distance \\
\hline LAR & Flat & Route Cache & Mobility based & No & $\begin{array}{l}\text { Shortest path route metric method is used. Multiple } \\
\text { routes are available in route reconfiguration } \\
\text { strategy, it erase route after source notification. }\end{array}$ \\
\hline GLS(Grid) & Flat & Two & $\begin{array}{l}\text { Mobility/Squares } \\
\text { based }\end{array}$ & Yes & $\begin{array}{l}\text { Queries success rate is less because queries are not } \\
\text { retransmitted, Success on the first try. }\end{array}$ \\
\hline GPSAL & Flat & Multiple & $\begin{array}{l}\text { Mobility/Squares } \\
\text { based }\end{array}$ & No & $\begin{array}{l}\text { Shortest path route metric method is used. In route } \\
\text { reconfiguration strategy it use alternate route or } \\
\text { back track until a route is found. }\end{array}$ \\
\hline
\end{tabular}

Below Table 4.2 provide the complexity comparison of geographical location based routing protocols, but the performance metrics represent the worst case scenario for each routing protocol. From this table we can know the average time complexity, memory overhead and control overhead of the five geographical locations based routing protocols.

Table 4.2: Comparisons of Complexities of Geographical location based routing protocols.

\begin{tabular}{|l|l|l|l|l|}
\hline Protocol & $\begin{array}{l}\text { Convergence } \\
\text { Time }\end{array}$ & $\begin{array}{l}\text { Memory } \\
\text { Overhead } \\
(\text { Mo })\end{array}$ & $\begin{array}{l}\text { Control } \\
\text { Overhead } \\
(\mathbf{C o})\end{array}$ & Advantages/Disadvantages \\
\hline DREAM & $\mathrm{O}(\mathrm{N}, \mathrm{I})$ & $\mathrm{O}(\mathrm{N})$ & $\mathrm{O}(\mathrm{N})$ & Low Co and Mo/requires a GPS. \\
\hline LAR & $\mathrm{O}(2 \mathrm{~S})$ & $\mathrm{O}(2 \mathrm{~S})$ & $\mathrm{O}(2 \mathrm{M})$ & $\begin{array}{l}\text { Localized route discovery/Based on source routing, flooding is used if no } \\
\text { location information is available. }\end{array}$ \\
\hline GLS(Grid) & $\mathrm{O}\left(\mathrm{Log}_{4} \mathrm{~N}\right)$ & $\mathrm{O}(2 \mathrm{~N})$ & $\mathrm{O}(2 \mathrm{~N})$ & $\begin{array}{l}\text { Route discovery to be done by using location update packet and GLS } \\
\text { query packet fields/care must be taken not to consume too much } \\
\text { bandwidth with the updates and split the grid in half at each level rather } \\
\text { than in fourth, so the network must recruit only Log }{ }_{2} \mathrm{~N} \text { location servers. }\end{array}$ \\
\hline GPSAL & $\mathrm{O}(\mathrm{D}+\mathrm{P})$ & $\mathrm{O}(\mathrm{N}+\mathrm{R})$ & $\mathrm{O}(\mathrm{A})$ & $\begin{array}{l}\text { Low overhead, small control packet size/flooding based route discovery } \\
\text { process. }\end{array}$ \\
\hline ZHLS & Intra : O(I) & $\mathrm{O}(\mathrm{I})+\mathrm{O}(\mathrm{D})$ & $\mathrm{O}(\mathrm{N} / \mathrm{M})^{\mathrm{a}}$ & $\begin{array}{l}\text { Reduction of SPF (Single Point of Failure), low Co/static zone map } \\
\text { required. }\end{array}$ \\
\hline
\end{tabular}

In DREAM, $\mathrm{N}$ is the number of nodes in the network and I indicate the fixed number of table updates required for transmission.

In LAR, $\mathrm{S}$ is the diameter of the nodes in the localized region and $\mathrm{M}$ indicates the number of nodes in the localized region.

In GLS(Grid), $\mathrm{N}$ indicates the number of nodes in the network.
In GPSAL, D indicates the diameter of the network, $\mathrm{P}$ is the diameter of the directed path of the RREP(Request Reply Packet), $\mathrm{N}$ is the number of nodes in the network and $\mathrm{A}$ is the number of affected nodes.

In ZHLS, O(I) represents the order of periodic update interval in Intra Zone, $O(D)$ represents the order of periodic update interval in Inter Zone, $\mathrm{O}(\mathrm{N} / \mathrm{M}) \mathrm{a}$ represents the number of zones or clusters in the network where $\mathrm{a}$ is the fixed number of updates sent at a fixed interval. 


\section{PERFORMANCE METRICS AND ANALYSIS}

In summary, flat routing protocols do not scale well, because their updating procedure consumes a significant amount of network bandwidth. DREAM routing protocol has scalability potential since it has significantly reduced the amount of overhead transmitted through the network by exchanging the location information. The hierarchical routing protocols will scale better over flat routing, because they introduce a structure to the network that controls the amount of overhead transmitted through the network. The common advantage associated with hierarchical protocols is mobility management. All proactive routing protocols have the same routing cost while considering the worst case scenario because they follow similar route discovery and maintenance procedure. LAR has the same cost as the traditional flooding algorithm in the worst case scenario. GLS, GPSAL and ZHLS protocols have the potential to provide higher scalability than pure reactive or proactive protocols because they attempt to minimize the number of rebroadcasting nodes by defining a structure which allows the nodes to work together in better organizing routing. The general factors of geographical location based routing protocols are illustrated in Table 5.1. The below graphs represents the comparisons of performance metrics like data packet delivery ratio, end to end delay, control packet overhead and total packet transmission for all the five geographical location based routing protocols.

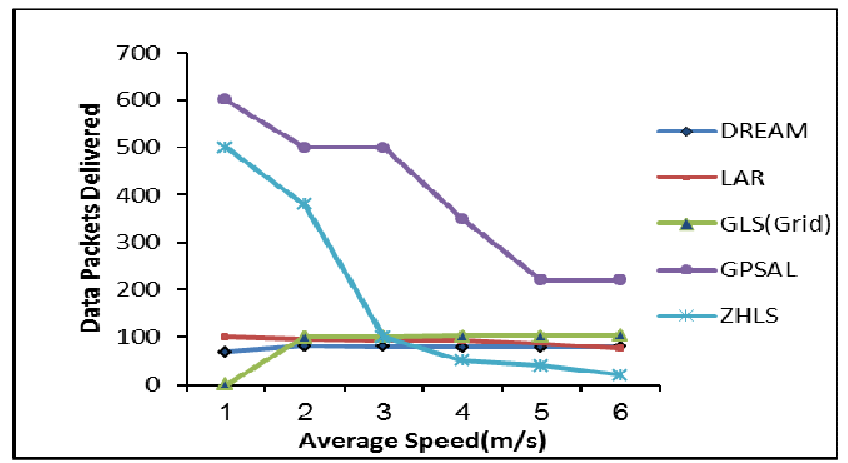

Chart -1: Data packet delivery ratio vs. speed

In DREAM protocol data messages are considered two orders of magnitude larger than control messages. Therefore data packets transmission is longer. The rate of transmission of each node is considered uniform all over the network. In LAR the number of routing packets and data packets are in an average speed. This is calculated as the ratio of number of routing packets and number of data packets received by the destination. These are constantly lower for LAR compared to flooding. As the speed of mobile hosts is increased the number of routing packets begins to increase for all routing protocols. In LAR the number of route requests is reduced by limiting route discovery to a smaller request zone. In GLS (Grid) the data traffic is generated by a number of constant bit rate connections equal to half the number of nodes. No node is a source in more than one connection and no node is a destination in more than three connections. Most of the data packets that grid fails to deliver are due to GLS query failure. These packets never leave the source. Once Grid finds a location of a destination, data losses are unlikely since geographic forwarding adapts well to the motion of intermediate nodes. Grid does a better job over a large number of nodes, especially for large network. GPSAL converges the number of iterations and the number of ants decreases. The traffic increases as the number of data packets between hosts increases and vice versa. In ZHLS, it successfully performs delivery packets from source to destination, higher this value gives the better results. It provides an expected data route length. Number of hops remains same in both cases of normal flooding and gateway flooding.

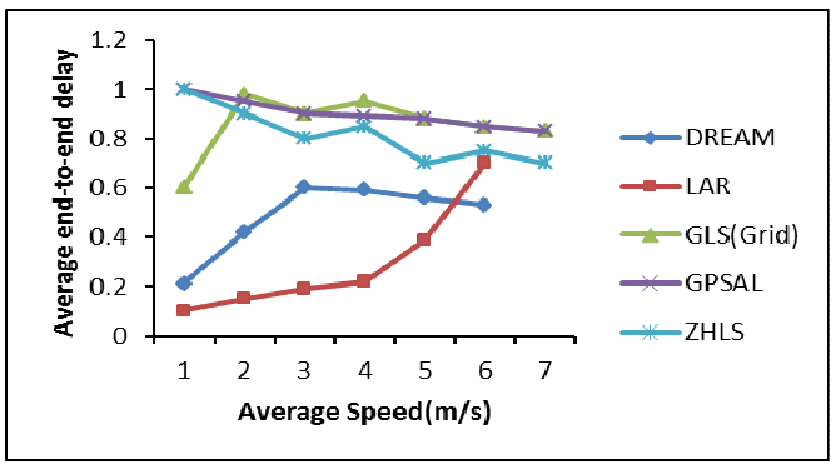

Chart -2: End-to-end Delay vs. speed

In DREAM the average end-to end delay of a data packet is obtained as speed increases. It is calculated from the first data packet to arrive at the destination. It has highest average end to end delay at speeds less than or equal to 10 $\mathrm{m} / \mathrm{s}$. at low speed it is accurate, due to contention and congestion in the network the data packet does not reach its intended destination. So it uses the recovery procedure. In LAR it only needs to do route discovery once at zero speed. It has a chance of sending data packets without the recovery procedure at zero speed. As speed increases more requests are needed thus slight increase in end-to-end delay compared to others, since LAR is able to use location information to focus its search for a route to a destination. LAR spends little time on route discovery at low speeds and at high speeds it spends time on route discovery. In GLS(Grid) end-to-end delay occurs because Grid fails to deliver due to GLS query failures. These packets never leave the source. Once Grid finds the location of a destination, data losses are unlikely and geographic forwarding adapts well to the motion of intermediate nodes. Grid does a better job over the whole range of number of nodes, especially for large networks. In GPSAL, end-to-end delays can be introduced while multiple nodes in a neighborhood attempt to transmit simultaneously. The traffic increases as the number of data packets between nodes also increases. In ZHLS there are possible delays caused by buffering during route discovery latency, queuing at the interface queue, retransmission delays at the MAC, propagation and transfer times. The average end-to-end delay is an average delay of data packets. It is also caused by queuing for transmission at the node and buffering data for detouring. The lower the end-to-end delay the better the application performance. Delay is less in gateway flooding. 


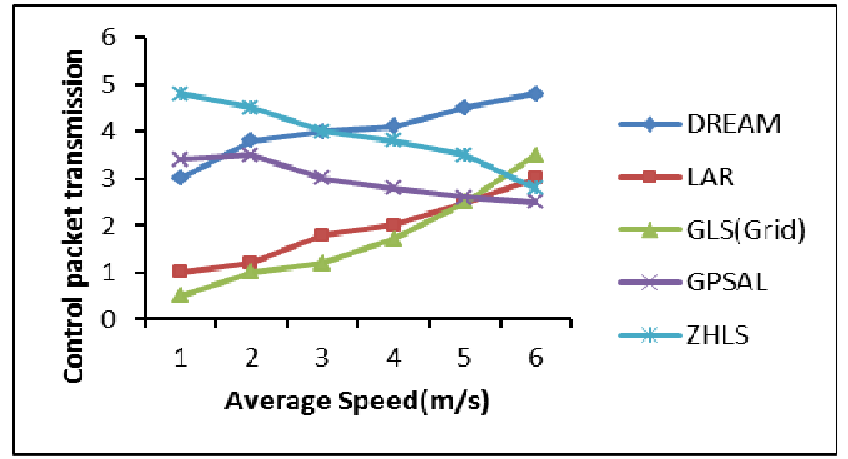

Chart -3: Control packet overhead vs. speed

The control packet overhead increses with inrease in mobilty as higher speed of nodes leads to more link failures which results in more route discoveries, thus increses the routing overhead. Control packets contain extra bytes to store hashes and intermediate node addresses.In DREAM it transmits many small control packets in its exchange of location information. Since DREAM returns an ACK for each data packet that is delivered from the forwarding zone, DREAM has the highest control packet overhead at low speeds. In LAR the route request is forwarded all the way to the destination before a response occur, thus LAR has the potential of transmitting more control packets. Control packet overhead of LAR increses substantially as speed increses since more route error and route request packets are transmited at higher speeds. In GLS(Grid), HELLO, GLS update and GLS query and reply packets are considered. Grid produces less overhead for large networks. Almost half of the route reply and cache reply messages are dropped due to congestion which causes more route requests into the network. Control overhead is considered interms of packets ratherthan bytes because medium acquisition overhead dominates actual packet transmission for the small packets used by Grid. In GPSAL overhead is present because of table exchanges and the introduction of ants. All tables are sent through broadcast. The value of tables sent means the amount of all routing information sent by all hosts where as tables received means the amount of routing information received. As expected the overhead is greater when ants are introduced.In ZHLS, it floods ZoneLSPs only to the gateway nodes of zones thus reduce the communication overhead significantly. In ZHLS, only the gateway nodes store ZoneLSPs and construct inter zone routing tables therefore the total storage capacity required in network is less than ZHLS. In ZHLS a gateway flooding scheme has been proposed to reduce the number of control packets.

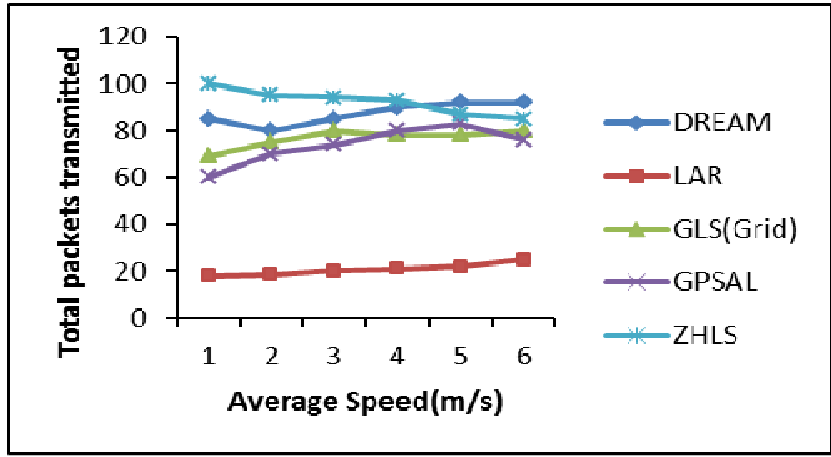

Chart -4: Total packets transmitted vs. speed

In DREAM always more than $80 \%$ of the data messages delivered have reached their final destination without restoring to a recovery procedure. Data packets are first flooded in the forwarding zone and then possibly flooded in the entire network. In this protocol recovery procedure is called very less. As the speed increases, DREAM remains constant due to the flooding behavior.LAR unicast the data packets. It has slightly higher data load for each data packet delivered at higher speeds. In LAR, while a node forwards a route request, it broadcasts the requests to all its neighbours with a samller transmission range. Number of neighbours for each node decreases. This factor decreases the probablity of a route discovery within the timeout interval using the initial route request zone. Because of this reason LAR do not perform too well when transmission range is small. Since LAR is based on restricted flooding the amount of packets increases exponentialy as the number of concurrent data packets are being routed. In GLS(Grid), total packets transmitted are considered by generating a number of constant bit rate connections equal to half the number of nodes. No node is a source in more than one connection and no node is a destination in more than three connections. Connections are inititaed at random times. As the speed increases the total packets transmitted also increases. In GPSAL the total amount of packtes present are routed at the same time. GPSAL considers all traffic as packets(ants, data packets and table exchanges) of all nodes, even the nodes that did not participate in the data packet delilvery. In ZHLS the total packets transmitted is the ratio between the number of packets sent by the source node and the number of packets received by the destination node. ZHLS is better in terms of packet delivery ratio.

Table - 5.1 : General Factors of Geographical location based routing protocols.

\begin{tabular}{|l|l|}
\hline Routing Class & Reactive / Proactive \\
\hline Routing Structure & Flat / Hierarchical \\
\hline Availability of route & Determined when needed \\
\hline
\end{tabular}




\begin{tabular}{|l|l|}
\hline Control traffic volume & Lower than global routing and further improved using GPS \\
\hline Periodic updates & Not required but some nodes may require \\
\hline Handling effects of mobility & Yes \\
\hline Storage requirements & Depends on the number of routes kept or required \\
\hline Delay level & Higher \\
\hline Scalability level & $\begin{array}{l}\text { Source routing protocols up to few hundred nodes. Point-to-point } \\
\text { may scale higher. Also depends on the level of traffic and the } \\
\text { levels of multihopping. }\end{array}$ \\
\hline
\end{tabular}

\subsection{Research issues and challenges}

This paper shows that there are many approaches to perform location-based packet forwarding using geographical location based routing protocols. However, there still exist a number of issues and problems that need to be addressed in future research. One of the geographical location based routing protocol LAR make it possible to have larger networks without scalability problems but they also offer attackers new opportunities specially that most protocols broadcast location information clearly allowing anyone within range to receive. Hence, node position can be altered, making other nodes believe that it is in a different position. This may make nodes believe that the attacker is the closest node to the destination and choose it as the next hop. Consequently, this attacker will be able to alter or drop packets. Thus, it is worth that more intensive work can be done to secure geographical location-based routing protocols to be able to defend against several attacks not only from malicious nodes, but also from the compromised ones. Finally, a mobile ad hoc network may consist of hundreds or even thousands of nodes. Security mechanisms should be scalable to handle such a large networks [21].

\section{CONCLUSION}

In this paper, the classification of routing according to network structure and geographical protocols are discussed in detail. The basic characteristics and complexity comparison of Geographical location based routing protocols are represented in tables 4.1 and 4.2. A general comparison of geographical location based routing protocols is represented in Table 5.1. In this paper an effort has been made to concentrate on the comparison of DREAM, LAR, GLS(GRID), GPSAL AND ZHLS location based routing protocols.

In DREAM routing protocol node only exchange location information rather than complete link state or distance vector information. In LAR routing protocol which also uses a GPS, the route request packets propagate in the request zone only. Here inter zone routing is also possible. In ZHLS routing protocol which lead to the gateway nodes for route discovery process. Inter zone route discovery, packets collaborate between nodes can help in maintaining routing information longer time. But a single routing protocol cannot perform best in all situations, so the choice of routing protocol should be done carefully according to the requirements of a specific application. The future research work is to propose an extension of the existing geographical location based routing protocol which will be better in terms of security issues.

\section{REFERENCES}

[1]. C. Perkins, "Ad hoc Networks," Addison-Wesley

[2]. M. Ilyas, "The Handbook of Ad Hoc Wireless

Networks," CRC Press, 2003.

[3]. C.K.Toh, "Ad Hoc Mobile Wireless Networks: Protocols and Systems," Prentice HallEnglewood Cliff,NJ

[4]. Blazevic. L, Le Boudec. J.Y,Giordano. S "A locationbased routing method for mobile ad hoc networks",Broadband Wireless LAN Group, STMicroelectron., Geneva, Switzerland,Mobile Computing, IEEE Transactions on 2011

[5]. A K Verma, Mayank Dave and R C Joshi, "Classification of Routing Protocols inMANET", at National Symposium on Emerging Trends in Networking \& MobileCommunication (NSNM-2003).

[6]. A. Perrig, Y-C Hu, and D. B. Johnson, "Wormhole Protection in WirelessAd Hoc Networks," Technical Report TR01-384, Dept. of ComputerScience, Rice University.

[7]. Yang, H., Luo, H., Ye, F., Lu, S., and Zhang, L. Security in mobile ad hocnetworks Challenge and solution.IEEE wireless communication.

[8]. H Deng, W. Li, and D. Agrawal, Routing Security in Wireless Ad Hoc Networks.IEEE Communications Magazine, 2002

[9]. A K Verma, Mayank Dave and R C Joshi, "Secure Routing in Mobile Networks: AReview," International J. of Systemics, Cybernetics and Informatics (IJSCI), ISSN09734864 (Peer reviewed and accepted).

[10]. Yi-Chun Hu, Adrian Perrig, "A Survey of Secure Wireless Ad HocRouting", IEEE Security and Privacy May/June 2004.

[11]. Kamal Kant, Lalit K. Awasthi ,"A Survey On Routing Protocols for Manets and Comparisons", IEEE Supported 
International Conference on Recent Trends in Soft Computing and Information Technology TSCIT-09, 2010.

[12]. A K Verma, Mayank Dave and R C Joshi, "Classification of Routing Protocols inMANET", at National Symposium on Emerging Trends in Networking \& MobileCommunication (NSNM-2003).

[13]. Stefano Basagni, Imrich Chlamtac,Violet R. Syrotiuk, Barry A.Woodward, “ A Distance Routinf effect Algorithm for Mobility(DREAM).

[14]. Young-Bae Ko and Nitin H. Vaidya, "Location-Aided Routing (LAR) in Mobile Ad Hoc Networks",Department of Computer Science Texas A\&M University College Station, TX 77843- 3112,youngbae,vaidya@cs.tamu.edu [15].W-H. Liao, Y-C. Tseng and J-P. Sheu, "GRID: A Fully Location-Aware Routing Protocol forMobile Ad Hoc Networks," Telecommunication Systems, Vol. 18, 2001.

[16].Camara,D.Loureiro,A.A.F,“A GPS/ant-like routing algorithm for ad hoc networks", Wireless Communications and Networking Confernce, 2000. WCNC. 2000 IEEE

[17].Zygmunt J. Haas and Marc R. Pearlman, "The Performance of QueryControl Schemes for the Zone Routing Protocol," In Proc. of the ACMSIGCOMM '98 Conference.

[18]. X. Hong, K. Xu and M. Gerla, "Scalable Routing Protocols for Mobile Ad Hoc Networks," IEEENetwork, July 2009.

[19]. Reza Shokri, Julien Freudiger and Jean-Pierre Hubaux, "A Unifed Framework for Location Privacy", LCA,EPFL,Switzerland, REPORT-148708 June 2010.

[20]. "Privacy and Security in Wireless Sensor and Ad Hoc Networks" Edited by Guohong Cao, Wensheng Zhang and Sencun Zhu.

[21]. H Deng, W. Li, and D. Agrawal, Routing Security in Wireless Ad Hoc Networks.IEEE Communications Magazine, 2008.

\section{BIOGRAPHIES}

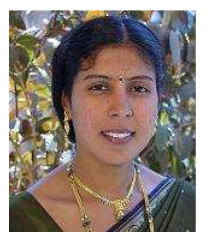

Suma $\mathbf{R}$ received B.Sc degree in Computer Science and Masters degree in Computer Applications from Bangalore University, Karnataka, India in 1995 and 1999 respectively.

She is pursuing her Ph.D. degree in Computer Science and Engineering at Visvesvaraya Technological University, Karnataka, India since 2011. She is currently working as an Associate Professor in the department of Master of Computer Applications at Sri Siddhartha Institute of Technology, Karnataka, India. She has 14 years of teaching experience in the area of Computer Science. Her research interests include Routing and Security in Mobile Ad Hoc Networks.

E-mail: sumaraviram@gmail.com

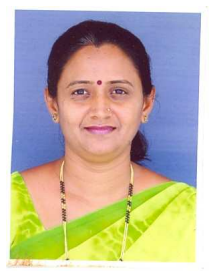

Smt. B.G.Premasudha received her B.E. (Electronics) degree from Bangalore University, Karnataka, India in 1990. Later in 1998, she obtained her MCA degree from Bangalore University.

She earned her doctoral degree from Dr.MGR University, Tamilnadu, India in the year 2010. Currently she is working as Professor in the Department of Master of Computer Applications, Siddaganga Institute of Technology, Tumkur, Karnataka, India. She has 23 years of teaching experience in the area of Computer Science. She had published many papers in several National and International conferences and Journals. She had won $3^{\text {rd }}$ prize for her paper at Second International conference and Indo-Canadian Satellite Symposium of Pharmaceutical Sciences, Technology and Health products held at Ooty, India during 24-26 Feb 2007. She had presented a paper at Map Middle East 2007 at Dubai during 9-11 April 2007 for which she had received travel grant from AICTE, India. Her areas of interest include Object Oriented Programming, GPS, GIS, Location Based Health Services and Wireless Communication and Networking. She had chaired several International and National conferences. She also serves on the advisory board of many colleges in Karnataka, India.

E-mail: bgpremasudha@gmail.com 